\title{
Incubation Period for Neuroinvasive Toscana Virus Infections
}

\author{
Lison Laroche, ${ }^{1}$ Frédéric Jourdain, ${ }^{1}$ Nazli Ayhan, Anne-Laure Bañuls, Rémi Charrel, Jorian Prudhomme
}

Toscana virus (TOSV) is an emerging pathogen in the Mediterranean area and is neuroinvasive in its most severe form. Basic knowledge on TOSV biology is limited. We conducted a systematic review on travel-related infections to estimate the TOSV incubation period. We estimated the incubation period at 12.1 days.

$\mathrm{T}$ oscana virus (TOSV) is an arthropodborne virus transmitted to humans through a bite from an infected sand fly (1). An RNA virus, it belongs to the genus Phlebovirus, species (Sandfly fever Naples phlebovirus family Phenuiviridae, order Bunyavirales) (2). TOSV infections are endemic to the Mediterranean basin and are considered frequent even though they are neglected (3). TOSV can be neuroinvasive and is a major cause of meningitis and encephalitis during summer months in areas to which it is endemic (4). However, most infections are asymptomatic or produce mild symptoms (5). Thus, TOSV cases are massively underestimated and unreported. Cases are mainly diagnosed by reverse transcription PCR in cerebrospinal fluid, blood, and, rarely, urine or by detecting virus-specific IgM or IgG (6). A total of 3 different TOSV lineages ( $\mathrm{A}, \mathrm{B}$, and $\mathrm{C})$ have been identified, but no clear evidence of a link between clinical manifestation and lineages exists (7).

In this study, we considered the incubation period (IP) of an infectious disease as the delay between infection and symptom onset; this definition differs from the latent period, which is defined as the time from infection to infectiousness. For arthropodborne viruses, the infectious bite represents the date of in-

Author affiliations: Université de Montpellier, Institut de Recherche pour le Développement, Centre National de la Recherche Scientifique, Montpellier, France (L. Laroche, F. Jourdain, A.-L. Bañuls, J. Prudhomme); Santé Publique France, Saint-Maurice, France (F. Jourdain); Aix-Marseille Université, Institut de Recherche pour le Développement, Institut national de la santé et de la recherche médicale, Marseille, France (N. Ayhan, R. Charrel)

DOI: https://doi.org/10.3201/eid2712.203172 fection (8). The potential period of exposure is represented by the length of stay in the country of infection before symptom onset. We therefore focused on imported cases.

Determining the IP is primordial for disease surveillance, outbreak investigation, public health interventions, infectious disease control, and modeling (9). However, IP estimates are often unsourced, imprecise, and based on limited evidence, as illustrated by the heterogeneous values proposed (Appendix Table 1, https://wwwnc.cdc.gov/EID/article/27/12/203172-App1.pdf). In this context, we conducted a systematic review of symptomatic travel-related neuroinvasive forms of TOSV to provide an evidence-based estimate of the IP.

\section{The Study}

We used PubMed and ISI Web of Knowledge search engines with no restriction on language and the phrase "Toscana AND virus AND (case report OR case-report OR travel* OR import*)." We conducted a systematic search on ProMed and Google Scholar, as well as cross-reference checking. The inclusion criteria were laboratory-documented acute TOSV infection, indication of a travel-related infection in a TOSVendemic area, and number of days between travel return and symptom onset. Two reviewers screened titles, abstracts, and full-text articles independently.

We extracted clinic and biologic elements from neuroinvasive TOSV case reports. For each patient, data related to the duration of travel and the time of symptom onset, gender, age, country in which case was reported, and country of infection were reported.

To estimate the IP, we used censored time-toevent models (10). Interval-censored observations related to travel duration represented the exposure time. Absence of a departure date was treated as left-censored data, whereas onset of illness during the travel period was considered right-censored. We performed data analysis by using $\mathrm{R}$ with the icenReg

\footnotetext{
${ }^{1}$ These authors contributed equally to this article.
} 
package ( $\mathrm{R}$ Foundation for Statistical Computing, https:/ / www.r-project.org). We defined the data distribution with 4 parametric models (log-normal, loglogistic, Gamma, and Weibull). To determine the best model for our distribution, we calculated the Akaike information criterion. We used the nonparametric log-rank test from the interval package to assess the effect of age and gender as covariates. To check the result stability, we performed an additional Bayesian approach, fitting with the Weibull distribution.

Regarding imported case reports, 142 documents were identified on PubMed and 133 on Web of Knowledge. We removed 79 duplicates and excluded 118 records after screening titles and abstracts. A total of 42 articles were eligible after full-text reading. We then selected 22 documents for data extraction. A total of 24 cases were selected (Appendix Table 2, Figure). All travel-associated cases fulfilling the inclusion criteria were neuroinvasive; these cases were diagnosed in a non-TOSV-endemic area after a stay in a proven TOSV-endemic area (Figure 1).

We selected Weibull distribution because it presented the lower Akaike information criterion (Figure 2; Appendix Table 3). The median IP for neuroinvasive forms is estimated to be 12.1 (95\% CI 10.2-14.4) days. In 5\% of neuroinvasive cases, symptoms will develop by 6.8 (95\% CI 3.8-9.9) days after an infectious bite; symptoms will develop in $95 \%$ of cases by 16.8 (95\% CI 13.9-21.7) days after the infectious bite. We found no evidence of age or gender effect on the length of the IP ( $p$ value $>0.05$ by log-rank test). By using Bayesian analysis, we found an IP of 12.1 (95\% CI 9.9-14.4) days (data not shown; results same as Figure 2).

\section{Conclusions}

In the literature, the IP values of TOSV are often heterogeneous, unsourced, or without evidence and therefore do not constitute a valid estimate for clinical or infection control decisions. Our literature review identified 24 neurologic cases of TOSV infection. Some travel durations were reported approximately in case reports and were not included in the analysis. All the data used were based on severe neurologic forms of the disease, which required hospitalization soon after the exposure period.

We estimated the median IP of TOSV at 12 (95\% CI 10.2-14.4) days. Considering the delay from infection to symptom onset, this value is greater than that for most other arboviruses (11). Our estimate of the IP is evidence-based and relies on data from well-characterized cases. However, cases that cause mildersymptoms, as opposed to neuroinvasive forms of the disease, might have a shorter IP (similar to

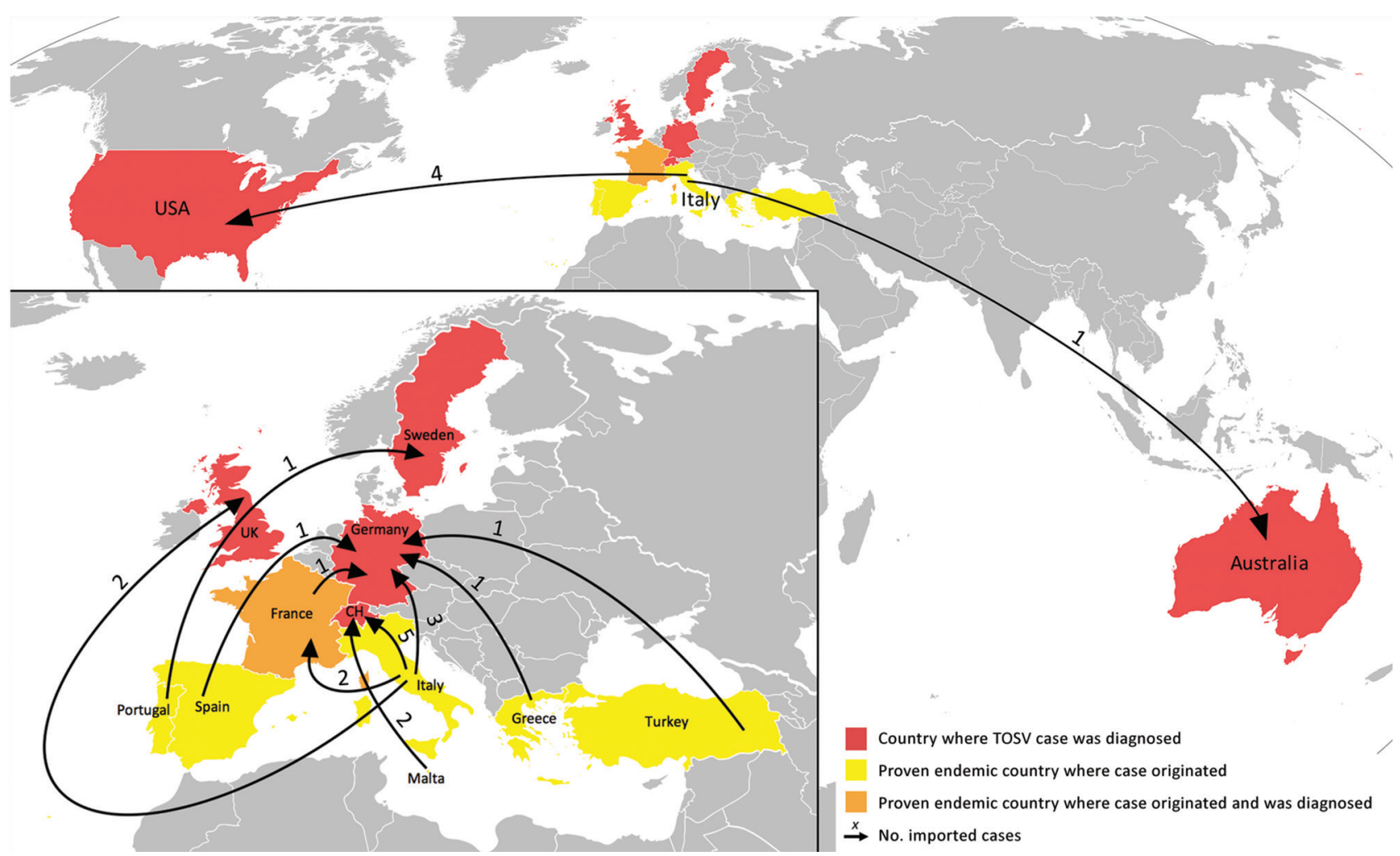

Figure 1. Geographic distribution of imported neuroinvasive cases of TOSV ( $n=24)$ and countries of origin of infection. TOSV, Toscana virus. 


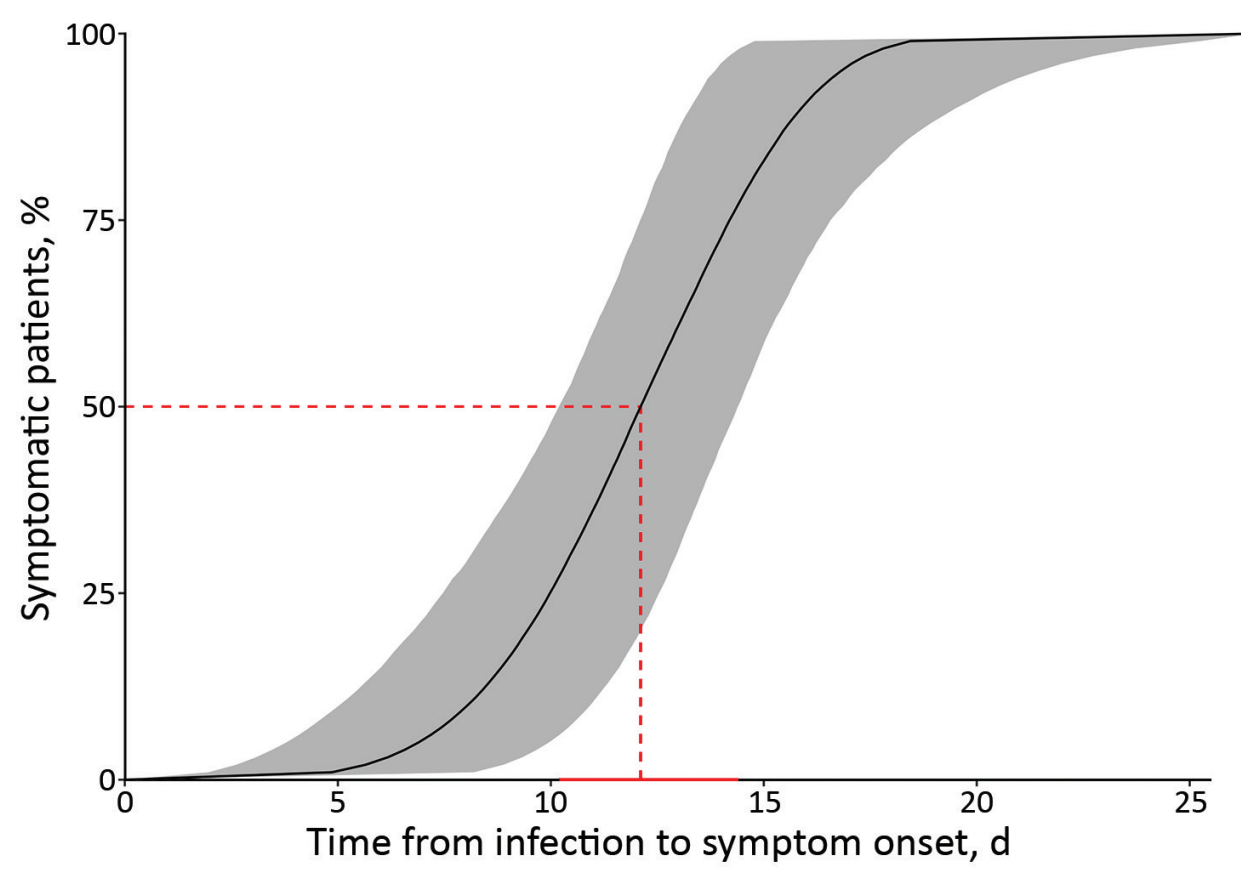

Figure 2. Cumulative percentage of Toscana virus cases manifesting with neurologic symptoms by a given day under the estimates for the Weibull parametric distribution $(n=24)$. Red dashed line represents the median estimation of the incubation period. Solid red horizontal line represents the $95 \% \mathrm{Cl}$ of the median. Gray shading indicates the $95 \% \mathrm{Cl}$ of the values. other arboviruses). Other symptoms associated with paucisymptomatic forms of TOSV might not have been described yet and should be further investigated to improve case definition and diagnosis.

We also cannot exclude infections by other sandfly fever Naples phleboviruses because of crossreaction risk in serologic analyses due to their close genetic relationships (12). However, the incidence in the population of other genetically similar phleboviruses is lower than TOSV, and TOSV remains the most common cause of neuroinvasive symptoms (3). Knowledge of TOSV genotypes and their aptitude to cause different clinical forms is limited (12). Analyzing this hypothesis was not possible because of the limited amount of available data. In addition to the genotype, other parameters may influence the IP, such as viral strain, patient's immune status, or viremia (9). The amount of virus transmitted during bites (viral load) could also influence the IP and should be further investigated.

In addition, all other cases were diagnosed in countries or regions to which TOSV is not endemic (United States, United Kingdom, Sweden, Germany, Switzerland, Australia, and France). These imported cases represent a risk for emergence in these areas when vectors are established (13), as has been observed for other vectorborne diseases (14). Moreover, sand flies are known to spread in countries or regions to which TOSV is not endemic (15).

Currently, information on TOSV infections is lacking (12). Precise definitions of the IP should provide more information on the disease epidemiology and on its development in the human host. Moreover, because the IP is a key parameter for disease modeling (9), it would improve our understanding of the disease transmission dynamics. More reports of travel-related cases and standardization of data collection with reliable information (e.g., location and duration of the trips and precise dates of symptom onset) are clearly needed. The IP estimation will be improved with addition of new data.

\section{Acknowledgments}

We thank Julian Wei-Tze Tang, James Farrugia, Jonas Schmidt-Chanasit, Josef Georg Heckmann, and David Breen for answering our questions by email to clarify information regarding the TOSV case reports. We also thank

Eric Elguero for his support in statistical analysis, Heïdi Lançon for the English revision of the manuscript, and Jérôme Depaquit for reviewing the manuscript.

This research was funded by the IRD (Institut de Recherche pour le Développement), CNRS (Centre National de la Recherche Scientifique), UM (Université de Montpellier) and by the by the European Virus Archive Global (EVA-GLOBAL) project that has received funding from the European Union's Horizon 2020-INFRAIA-2019 research and innovation program under grant agreement No 871029. F.J. is financially supported by the SPF (Santé Publique France) through a doctoral fellowship, J.P. by the INFRAVEC2 project (https:/ /infravec2.eu), L.L. by a UM doctoral fellowship, and N.A. by a postdoctoral fellowship from the AMU (Aix Marseille University). 


\section{About the Author}

Ms. Laroche is a doctoral student at the French National Research Institute for Sustainable Development and the University of Montpellier, France. Her primary research interests are medical entomology, especially the interactions between pathogens (Phlebovirus, Leishmania), and their vector (the sand fly).

\section{References}

1. Depaquit J, Grandadam M, Fouque F, Andry PE, Peyrefitte C. Arthropod-borne viruses transmitted by Phlebotomine sandflies in Europe: a review. Euro Surveill. 2010;15:19507. https:/ / doi.org/10.2807/ese.15.10.19507-en

2. Ayhan N, Prudhomme J, Laroche L, Bañuls AL, Charrel RN. Broader geographical distribution of toscana virus in the Mediterranean region suggests the existence of larger varieties of sand fly vectors. Microorganisms. 2020;8:e114. https://doi.org/10.3390/microorganisms8010114

3. Charrel RN, Bichaud L, de Lamballerie X. Emergence of Toscana virus in the Mediterranean area. World J Virol. 2012;1:135-41. https://doi.org/10.5501/wjv.v1.i5.135

4. Charrel RN, Gallian P, Navarro-Marí JM, Nicoletti L, Papa A, Sánchez-Seco MP, et al. Emergence of Toscana virus in Europe. Emerg Infect Dis. 2005;11:1657-63. https:/ / doi.org/ 10.3201/eid1111.050869

5. Braito A, Corbisiero R, Corradini S, Marchi B, Sancasciani N, Fiorentini C, et al. Evidence of Toscana virus infections without central nervous system involvement: a serological study. Eur J Epidemiol. 1997;13:761-4. https:/ / doi.org/ 10.1023/A:1007422103992

6. Ergunay K, Kaplan B, Okar S, Akkutay-Yoldar Z, Kurne A, Arsava EM, et al. Urinary detection of toscana virus nucleic acids in neuroinvasive infections. J Clin Virol. 2015;70:89-92. https://doi.org/10.1016/j.jcv.2015.07.297

7. Ayhan N, Charrel RN. Of phlebotomines (sandflies) and viruses: a comprehensive perspective on a complex situation. Curr Opin Insect Sci. 2017;22:117-24. https:/ / doi.org/ 10.1016/j.cois.2017.05.019

8. Pingen M, Bryden SR, Pondeville E, Schnettler E, Kohl A, Merits A, et al. Host inflammatory response to mosquito bites enhances the severity of arbovirus infection. Immunity. 2016;44:1455-69. https://doi.org/10.1016/j.immuni.2016.06.002

9. Chan M, Johansson MA. The incubation periods of Dengue viruses. PLoS One. 2012;7:e50972. https://doi.org/10.1371/ journal.pone.0050972

10. Cowling BJ, Muller MP, Wong IOL, Ho LM, Louie M, McGeer A, et al. Alternative methods of estimating an incubation distribution: examples from severe acute respiratory syndrome. Epidemiology. 2007;18:253-9. https:// doi.org/10.1097/01.ede.0000254660.07942.fb

11. Rudolph KE, Lessler J, Moloney RM, Kmush B, Cummings DAT. Incubation periods of mosquito-borne viral infections: a systematic review. Am J Trop Med Hyg. 2014;90:882-91. https://doi.org/10.4269/ajtmh.13-0403

12. Maes P, Adkins S, Alkhovsky SV, Avšič-Županc T, Ballinger MJ, Bente DA, et al. Taxonomy of the order Bunyavirales: second update 2018. Arch Virol. 2019;164:92741. https:// doi.org/10.1007/s00705-018-04127-3

13. Knechtli R, Jenni L. Distribution and relative density of three sandfly (Diptera: Phlebotominae) species in southern Switzerland [in French]. Ann Parasitol Hum Comp. 1989;64:53-63. https://doi.org/10.1051/parasite/198964153

14. Liang G, Gao X, Gould EA. Factors responsible for the emergence of arboviruses; strategies, challenges and limitations for their control. Emerg Microbes Infect. 2015;4:e18. https://doi.org/10.1038/emi.2015.18

15. Maroli M, Feliciangeli MD, Bichaud L, Charrel RN, Gradoni L. Phlebotomine sandflies and the spreading of leishmaniases and other diseases of public health concern. Med Vet Entomol. 2013;27:123-47. https://doi.org/10.1111/ j.1365-2915.2012.01034.x

Address for correspondence: Lison Laroche, UMR MIVEGEC, IRD-CNRS-Université de Montpellier, 911 avenue Agropolis, F34394 Montpellier, France; email: lisonlaroche14@gmail.com 